\title{
AÇÕES DE PREVENÇÃO DE CÂNCER DE MAMA ENTRE DOCENTES DE ENFERMAGEM
}

Sayane Marlla Silva Leite Montenegro ${ }^{1}$, Maria Bernadete de Sousa Costa $^{2}$, Simone Helena dos Santos Oliveira ${ }^{3}$, Leila de Cássia da Fonseca ${ }^{4}$, José Melquiades Ramalho Neto ${ }^{5}$, Daniel Lima de Farias ${ }^{6}$

RESUMO: Trata-se de um estudo exploratório, descritivo, de natureza quantitativa, e teve por objetivo verificar ações de prevenção de câncer de mama entre 20 docentes de Enfermagem da Universidade Federal da Paraíba. Os dados foram coletados em agosto e setembro de 2011, os resultados revelaram que entre as participantes a faixa etária prevalente foi de 40 a 50 anos e que $60 \%$ desempenham atividade exclusiva de docência. Constatou-se que $17(85 \%)$ participantes realizam exames de prevenção contra o câncer de mama, principalmente por meio da ultrassonografia mamária e mamografia, embora apenas $45 \%$ realizem o autoexame mensalmente. Os resultados apontam que as docentes não realizam ações de prevenção do câncer de mama na totalidade, evidenciando uma lacuna entre o que preconizam como profissionais de saúde e o exercido como mulheres.

DESCRITORES: Enfermagem; Prevenção de Câncer de Mama; Atenção Primária à Saúde.

\section{ACTIONS FOR PREVENTION OF BREAST CANCER AMONG NURSING LECTURERS}

ABSTRACT: This quantitative, exploratory and descriptive study aimed to ascertain actions for the prevention of breast cancer among 20 nursing lecturers at the Federal University of Paraíba. The data was collected in August and September 2011, and the results showed that among the participants, the predominant age range was $40-50$ years old, and that $60 \%$ worked exclusively as lecturers. It was confirmed that $17(85 \%)$ participants undertake examinations for prevention of breast cancer, principally through ultrasound of the breast and mammography, but that only $45 \%$ carry out monthly selfexamination. The results indicate that the lecturers do not undertake the full range of measures against breast cancer, evidencing a gap between what they recommend as health professionals and what they do, as women.

DESCRIPTORS: Nursing; Prevention of breast cancer; Primary health care.

\section{ACCIONES DE PREVENCIÓN DE CÁNCER DE MAMA ENTRE DOCENTES DE ENFERMERÍA}

RESUMEN: Este es un estudio exploratorio, descriptivo, de naturaleza cuantitativa, cuyo objetivo fue verificar acciones de prevención de cáncer de mama entre 20 docentes de Enfermería de la Universidad Federal de Paraíba. Los datos fueron recogidos en agosto y septiembre de 2011, y los resultados revelaron que entre las participantes la franja etaria más frecuente fue de 40 a 50 años y que $60 \%$ desempeñan actividad exclusiva de docencia. Se constató que 17(85\%) participantes realizan exámenes de prevención contra el cáncer de mama, principalmente por medio de ecografía mamaria y mamografía, a pesar de solamente $45 \%$ realizar el examen en sí misma mensualmente. Los resultados apuntan que las docentes no realizan acciones de prevención del cáncer de mama en la totalidad, evidenciando una laguna entre lo que preconizan como profesionales de salud y el ejercido como mujeres.

DESCRIPTORES: Enfermería; Prevención de Cáncer de Mama; Atención Primaria a la Salud.

\footnotetext{
${ }^{1}$ Enfermeira Residente do Hospital Universitário Lauro Wanderley da Universidade Federal da Paraíba - UFPB.

${ }^{2}$ Enfermeira. Doutora em Enfermagem. Professora do Curso de Graduação em Enfermagem da UFPB.

${ }^{3}$ Enfermeira. Doutora em Enfermagem. Professora do Curso de Graduação em Enfermagem da UFPB e da Escola Técnica em Saúde. ${ }^{4}$ Enfermeira. Mestre em Enfermagem. Professora do Curso de Graduação em Enfermagem da UFPB.

${ }^{5}$ Enfermeiro Assistencial da Unidade de Terapia Intensiva Geral do Hospital Universitário Lauro Wanderley da UFPB. Mestre em Enfermagem. Professor do Curso de Graduação em Enfermagem da Faculdade de Enfermagem Nova Esperança - João Pessoa-PB.

${ }^{6}$ Biólogo. Especialista em Gestão em Saúde e Pedagogia do Campo. Mestrando pelo Programa de Pós-Graduação em Biologia Molecular e Celular pela UFPB.
}

Autor correspondente:

Sayane Marlla Silva Leite Montenegro

Universidade Federal da Paraíba

R. Dineza Carneiro Monteiro, 58 - 58055-710 - João Pessoa-PB-Brasil

E-mail: sayane_ufpb@hotmail.com
Recebido: $18 / 05 / 2012$ Aprovado: 24/10/2012 


\section{INTRODUÇÃO}

O presente estudo emergiu a partir da divulgação dos elevados índices de morbimortalidade do câncer de mama e de colo de útero no Brasil, publicados em 2008 , que apontava para 49.400 novos casos. Na capital do Estado da Paraíba, cenário deste estudo, entre os anos 2001 e 2005 foram registrados 965 novos casos de câncer de mama, destacando-se maior incidência na faixa etária entre 40 e 55 anos $^{(1)}$.

A palavra cuidar tem sua origem do latim cogitare (cogitar). Significa que o cuidar é acima de tudo aplicar atenção a alguma coisa ou a alguém, assim como aplicar o pensamento e a imaginação ${ }^{(2)}$. Cuidar para o enfermeiro, por muitas vezes, se parece uma metáfora do dia-a-dia, pois cuidas faz parte de seu cotidiano. $\mathrm{O}$ enfermeiro cuida de todos, seja do paciente, dos seus familiares e dos amigos, daí a definição de profissão do cuidado, mas é preciso rever como essa profissão tomou tal dimensão ${ }^{(3)}$.

As práticas do cuidar, desde muito tempo existem, nessas a figura feminina está no centro, associada à sobrevivência e vigilância. Segundo autores, durante a difusão do Cristianismo a enfermagem era exercida por religiosas que relacionavam o cuidado a prática da caridade. No entanto, com a reforma religiosa, a enfermagem teve uma decadência, passando de cuidadoras religiosas para mulheres de pouco brilho social, isto é, a enfermagem era exercida por mulheres marginalizadas ${ }^{(3)}$.

Neste ensejo, pode-se considerar, além do cuidar por parte do profissional cuidador, o autocuidado, que representa atividade relevante e própria da pessoa, compreendida pelo mesmo e orientado para um objetivo, o cuidado com a própria saúde. $\mathrm{O}$ autocuidado foi evidenciado pela primeira vez em 1985, no campo da enfermagem, por Dorothea Elizabeth Orem, a qual teceu reflexões sobre o porquê dos indivíduos necessitarem do auxílio da enfermagem e poderem ser ajudados pela mesma ${ }^{(2)}$.

No que se refere ao autocuidado com as mamas, deve-se ressaltar que as primeiras descrições de tumores foram encontradas em papiros do Egito, e datam de 1.600 a.C. Somente no século XVIII o tabu da doença começou a mudar, com John Hunter, cirurgião escocês, que sugeriu que alguns cânceres poderiam ser curados por cirurgia. Nesse mesmo século, um médico italiano atentou para o fato de que os hormônios poderiam ter influência sob a doença. A partir desta época, a prevenção e tratamento das neoplasias se tornaram cada vez mais avançados e divulgados ${ }^{(3)}$.
Ainda referente ao câncer de mama, sabe-se que se o tumor não está limitado à mama, podendo comprometer linfonodos ou outras estruturas, a sobrevida em cinco anos é de $97 \%$. Em havendo comprometimento, esta taxa é de $78 \%$. Em doença avançada com tumores presentes em locais distantes do tumor primário, a sobrevida em cinco anos chega a $23 \%{ }^{(1)}$. Oportuna-se objetivar que os tumores de mama crescem em velocidades diferentes, mas oncologistas estimam que o tumor dobre de tamanho a cada 100 dias, daí a importância do autoexame das mamas para uma melhor palpação e reconhecimento de anormalidades mamárias ${ }^{(1)}$.

Diante desses fatos surgiram os seguintes questionamentos: As docentes do curso de graduação e pós-graduação em Enfermagem do Centro de Ciências da Saúde da Universidade Federal da Paraíba realizam ações de prevenção contra o câncer de mama? Quais ações relativas à prevenção do câncer são adotadas pelas docentes?

Para responder a tais questionamentos este estudo teve por objetivos verificar ações de prevenção do câncer de mama entre docentes do curso de graduação e pós-graduação em enfermagem da Universidade Federal da Paraíba.

\section{MÉTODO}

O estudo configurou-se como pesquisa exploratória descritiva, de natureza quantitativa, caracterizado como pesquisa de campo. A pesquisa exploratória tem a finalidade de familiarizar-se com o fenômeno ou obter uma nova percepção dele e descobrir novas ideias, esta não requer a elaboração de hipótese a serem testadas no trabalho, restringindo-se a definir objetivos e buscar mais informações sobre determinado assunto de estudo(4).

$\mathrm{O}$ estudo foi realizado entre os meses de janeiro e dezembro de 2011 na Universidade Federal da Paraíba, no Departamento de Enfermagem Clínica e no Departamento de Enfermagem em Saúde Pública, localizados no campus I, em João Pessoa-Paraíba.

A escolha da instituição deve-se ao fato de ser uma entidade destinada à formação de recursos humanos voltados para a área de saúde e outras áreas afins, como a Enfermagem, disponibilizando, Cursos de Graduação e Pós-Graduação em Enfermagem, além de Cursos Técnicos. A população do estudo foi constituída por todas as 20 professoras do Curso de Graduação e de Pós-Graduação em Enfermagem do Centro de Ciências da Saúde da Universidade Federal da Paraíba que desempenhavam atividadesna Universidade. 
A pesquisa foi conduzida levando-se em consideração os aspectos éticos da pesquisa realizada com seres humanos, obedecendo aos critérios estabelecidos na Resolução 196/96 $6^{(5)}$ e foi aprovada pelo Comitê de Ética em Pesquisa do Centro de Ciências da Saúde sob protocolo n. 061/2011.

A coleta de dados foi realizada nos meses de agosto e setembro de 2011, mediante a aplicação de questionário semiestruturado, contendo questões que compreenderam as variáveis do perfil das participantes: faixa etária, disciplina que leciona e outras atividades profissionais. E variáveis socioepidemiológicas tais como: realização de ações de prevenção do câncer de mama, tipos de ações e periodicidade com que realiza.

A coleta de dados foi realizada pela própria pesquisadora observando os seguintes passos: Contatar as professoras no campo da pesquisa explicando os objetivos e a importância da sua participação no estudo; Agendar horário e local das entrevistas conforme a disponibilidade de cada participante; Solicitar assinatura do Termo de Consentimento Livre e Esclarecido; e Esclarecer possíveis dúvidas quanto ao preenchimento do instrumento.

Inicialmente, foi efetuada a definição e a codificação das variáveis pertinentes ao estudo. Na sequência, utilizou-se a estatística descritiva e o software SPSS 15.0 para cálculo das frequências e percentuais de cada variável. Os resultados foram apresentados em quadros e tabelas, e discutidos com base na correspondente literatura científica.

\section{RESULTADOS}

A faixa etária dos participantes está aprensentada na tabela 1.

Constatamos que 17 (85\%) docentes realizam ações de prevenção contra o câncer de mama, no entanto 3 (15\%) referiram não realizar, entre essas duas ministram a disciplina Saúde da Mulher e uma a disciplina Enfermagem Cirúrgica.

Com relação às ações, a tabela 2 apresenta o tipo de prevenção, com destaque para os exames de ultrassonografia mamária e mamografia e para o exame médico. Contudo, com relação à periodicidade, 14 (70\%) referiram ter realizado os exames em 2011, 4 $(20 \%)$ realizaram no último ano e $2(10 \%)$ docentes referiram ter realizado há muitos anos atrás.

Entre as docentes que referiram realizar o autoexame das mamas, $9(75 \%)$ o fazem mensalmente.
Tabela 1 - Distribuição das docentes segundo faixa etária. João Pessoa, 2011

\begin{tabular}{lcc}
\hline Faixa etária (anos) & $\mathbf{n}$ & $\mathbf{\%}$ \\
\hline Menos de 30 & 2 & 10,0 \\
Entre 31 e $<40$ & 6 & 30,0 \\
Entre 40 e 50 & 8 & 40,0 \\
Mais de 50 & 4 & 20,0 \\
Total & 20 & 100,0 \\
\hline
\end{tabular}

Tabela 2- Ações de prevenção contra o câncer de mama entre docentes de enfermagem. João Pessoa, 2011

\begin{tabular}{lcc}
\hline AÇõES DE PREVENÇÃO & $\mathbf{n}$ & $\mathbf{\%}$ \\
\hline Utrassonografia mamária & & \\
Sim & 15 & 75,0 \\
Não & 5 & 25,0 \\
Autoexame & & \\
Sim & 12 & 60,0 \\
Não & 8 & 40,0 \\
Mamografia & & \\
Sim & 15 & 75,0 \\
Não & 5 & 25,0 \\
Consulta Médica & & \\
Sim & 15 & 75,0 \\
Não & 5 & 25,0 \\
\hline
\end{tabular}

\section{DISCUSSÃO}

Ao analisar a tabela 1, observa-se que a maioria dos participantes se encontra na faixa etária de 40 a 50 anos. Autores afirmam que a idade é um dos fatores de risco para o câncer de mama ${ }^{(6)}$; partindo deste pressuposto, a faixa etária mais prevalente em nosso estudo está situada entre as de maior incidência desta neoplasia. Pouco menos de 5\% dos casos ocorrem em mulheres abaixo de 30 anos, porém estas estatísticas tendem a aumentar na faixa etária mais jovem devido a maus hábitos alimentares e práticas sexuais com vários parceiros, favorecendo a instalação de problemas indesejáveis $^{(7-9)}$.

A tabela 2 nos revela que a maioria dos docentes realiza ações de prevenção que realizam.

De acordo com as recomendações, as ações estão centradas na prevenção, detecção precoce e transmissão de informação com qualidade ${ }^{(10)}$. Neste sentido, no momento em que estas ações são realizadas as chances de detectar alterações precocemente são maiores. Torna-se imprescindível destacar que o câncer de mama tem 95\% de chance de cura quando detectado no início. Pesquisa revelou que cerca de $60 \%$ das vezes, o tumor de mama é 
detectado tardiamente, sem expectativa de cura, levando em consideração que as ações de prevenção foram mascaradas $^{(11)}$.

Observa-se que nas respostas das docentes estão presentes duas ações para prevenção do câncer de mama, a utrassonografia mamária e a mamografia. $\mathrm{O}$ exame imagenológico apresenta elevadas sensibilidade (77 a 95\%) e especificidade (94 a 97\%), sendo assim o rastreamento mamográfico reduz a mortalidade do câncer de mama em 15\% para mulheres entre 39 e 49 anos; mulheres mais jovens apresentam mais mamografias falso-positivas devido às alterações mamárias que ainda estão em atividade ${ }^{(12-14)}$.

A realização da prevenção por meio do autoexame implica conhecimento das mulheres sobre seu corpo, haja vista que a detecção de alguma anormalidade, no momento do autoexame, é facilitada quando as mulheres já apresentam certa intimidade com o próprio $\operatorname{corpo}^{(15)}$. Nos casos em que este procedimento não ocorre, o câncer acaba sendo descoberto num estágio mais avançado necessitando, muitas vezes, de intervenção invasiva, como a retirada de um quadrante da mama ou até mesmo de toda a mama.

A partir do já citado e do grande apego da mulher às suas mamas, pode-se objetivar que uma intervenção da magnitude de uma mastectomia, seja ela parcial ou total, pode trazer um desequilíbrio emocional muito grande na vida da mulher, visto que refere-se à mama, órgão que traz embutido, além da questão da saúde, aspectos ligados à feminilidade, beleza, sensualidade da mulher e principalmente maternidade ${ }^{(10)}$.

Neste estudo observou-se que as docentes de enfermagem preferem estratégias de prevenção mais caras e de difícil acesso, em detrimento à estratégia de prevenção, como o autoexame que é simples e acessível. $\mathrm{O}$ autoexame da mama é uma forma de prevenção e detecção precoce de alterações mamárias e deve ser realizado mensalmente entre o $7^{\circ}$ e $10^{\circ}$ dia antes ou depois do ciclo menstrual regular. Para as mulheres que apresentam irregularidades menstruais, o autoexame deve ser realizado do $7^{\circ}$ ao $10^{\circ}$ dia após a menstruação. As mulheres histerectomizadas, na menopausa ou que estão amamentando indica-se estabelecer um dia no mês para realizar o autoexame ${ }^{(16)}$.

A partir dos 35 anos é indicado, além do autoexame das mamas a mamografia, com intervalo máximo de dois anos entre cada exame em mulheres que nunca apresentaram alterações mamárias. Em mulheres que já apresentaram alguma alteração mamária o período de realização é reduzido para um ano ${ }^{(11-13)}$.

\section{CONCLUSÃO}

Os resultados obtidos neste estudo mostram que as docentes participantes estão entre as faixas etárias de maior incidência do câncer de mama, que a maioria adota autoexame das mamas e outras práticas preventivas, por meio de tecnologias, como mamografia e ultrassom. Contudo, este estudo possibilita reflexões acerca da enfermidade que constitui, na atualidade, sério problema de saúde pública. Neste contexto, mesmo pessoas com alto nível de instrução e formadoras de profissionais de saúde, podem não realizar a prevenção em sua totalidade, ressaltando uma lacuna entre o preconizado como profissional de saúde e o exercido como mulher.

\section{REFERÊNCIAS}

1. Brasil. Instituto Nacional de Câncer. Coordenação dos Programas de Controle do Câncer/Pro-Onco. Estimativas da incidência e mortalidade por câncer no Brasil para 2008 e 2009. Brasília: Ministério da Saúde; 2007.

2. Orem D. Nursing: concepts of practice. $6^{\mathrm{a}}$ ed. St. Louis: Mosby; 2001.

3. Ribeiro ET. Curso de rotinas práticas de direito e de processo do trabalho. $3^{\mathrm{a}}$ ed. Porto Alegre: Síntese; 2002.

4. Cervo AL, Bervian PA, Silva R. Metodologia Científica. São Paulo: Pearson Prentice Hall; 2006.

5. Brasil. Resolução n 196/96 dispõe sobre Diretrizes e Normas Regulamentadoras de Pesquisa envolvendo Seres Humanos. Diário Oficial da União. Conep;1996.

6. Brasil. Secretaria de Vigilância em Saúde. Situação da prevenção e controle das doenças transmissíveis no Brasil. Brasília: Ministério da Saúde; 2010.

7. Menke $\mathrm{CH}$ Rotinas em mastologia. Porto Alegre: Art. Med; 2000.

8. Nasajon LW, Balem JL. A evolução do câncer de mama na mulher jovem - uma revisão da literatura. Femina. 1999;27(9):745-7.

9. Tessaro S. Contraceptivos orais e câncer de mama: estudo de casos e controles. Rev. Saúde Públ. 2001;35(1):35-8.

10. Marinho MS, Costa - Gurgel LAB, Cecatti JG, Osis MJD. Conhecimento, atitude e prática do autoexame das mamas em centros de saúde. Rev. Saúde Públ. 2005;37(5):576-82. 
11. Novais E. Toque não evita morte por câncer de mama [Internet] [acesso em 12 ago 2011]. Disponível: http:// www.netsite.com. artigo/mostra_conteudo121416. asp?id_conteudo $=121416>$

12. Davim RMB, Torres GV, Cabral MLN. Breast selfexamination: the knowledge of users assisted in the outpatient unit of a university maternity hospital. Rev. Latino-Am. Enfermagem. 2003;11(1):21-7.

13. Instituto Nacional de Câncer-INCA. Coordenação dos programas de controle do câncer/Pro-Onco. Estimativas da incidência e mortalidade por câncer no Brasil para 2008 e 2009. Brasília: Ministério da Saúde; 2007.

14. Nelson HD, Tyne K, Naik A, Bougatsos C, Chan BK, Humphrey L. Screening for breast cancer: systematic evidence review. Ann Intern Med. 2009;151(10):727-42.

15. Laganá MTC, Gualda MD, Hashimoto RM, Imanichi MR. Auto-exame de mama: identificação dos conhecimentos, atitudes, habilidades e práticas requeridas para elaboração de propostas educativas. Rev Esc Enferm USP. 1990;24(2),281-99.

16. Marinho MS, Costa-Gurgel LAB, Cecatti JG, Osis MJD. Conhecimento, atitude e prática do autoexame das mamas em centros de saúde. Rev. Saúde Públ. 2003;37(5):576-82. 